\title{
Editorial: Revisiting the Effectiveness of Transcranial Direct Current Brain Stimulation for Cognition: Evidence, Challenges, and Open Questions
}

\author{
Evangelia G. Chrysikou ${ }^{1 *}$, Marian E. Berryhill ${ }^{2}$, Marom Bikson ${ }^{3}$ and H. Branch Coslett ${ }^{4}$ \\ ${ }^{1}$ Department of Psychology, University of Kansas, Lawrence, KS, United States, ${ }^{2}$ Department of Psychology, University of \\ Nevada, Reno, NV, United States, ${ }^{3}$ Department of Biomedical Engineering, City University of New York, New York, NY, United \\ States, ${ }^{4}$ Department of Neurology, University of Pennsylvania, Philadelphia, PA, United States
}

Keywords: transcranial direct current stimulation (tDCS), neuroenhancement (NE), noninvasive brain stimulation, cognition and emotion, neurorehabilitation

\section{Editorial on the Research Topic}

Revisiting the Effectiveness of Transcranial Direct Current Brain Stimulation for Cognition: Evidence, Challenges, and Open Questions

Over the past 15 years, there has been an explosion of interest in the use of noninvasive brain stimulation approaches to study the brain. Some studies have suggested that transcranial Direct Current Stimulation (tDCS) in particular can elicit positive effects on performance for many aspects of cognition, including working memory, attention, executive function, language, and numerical competence. A growing literature further indicates that tDCS can provide potentially long-lasting benefits for patient rehabilitation, ameliorating wide-ranging conditions such as aphasia, pain, major depression, tinnitus, and migraine, among others.

It is well-accepted that tDCS is a well-tolerated, noninvasive technique that involves the application of low levels of direct current (1-2 mA, 10-30 min) through electrodes placed on the scalp to alter the neural activity of underlying neural populations. It is often assumed that during and immediately after application, cortical excitability increases under the anode electrode because of neuron soma depolarization, whereas cortical excitability decreases under the cathode electrode because of neuron soma hyperpolarization (Purpura and McMurtry, 1965; Nitsche and Paulus, 2000, 2001) - however, both the outcomes and mechanisms of tDCS are more complex (Giordano et al., 2017; Jamil et al., 2017; Kronberg et al., 2017). Long-term effects of tDCS have been linked to neuroplasticity following LTP-like changes in synaptic strength between stimulated neurons involved in task performance (Reato et al., 2010; Rahman et al., 2015). An advantage of the procedure relative to other brain stimulation techniques is its reliable sham manipulation: active tDCS is silent, does not induce muscle twitches, and it is not immediately distinguishable from sham stimulation, thus allowing for double blinded studies (but see Giordano et al., 2017). Critically, the existing availability of devices that can administer tDCS, its ease of use, and its excellent safety profile underscore the potential of tDCS as a tool for improving cognitive performance in healthy populations, stabilizing cognition in those who are at high risk for cognitive decline, and providing adjuvant therapy for those in need of cognitive rehabilitation.

Nevertheless, despite these recent advances in the use of the procedure, the precise neurobiological mechanisms underlying tDCS effects in humans remain insufficiently understood. Tempering the enthusiasm for this methodology, a number of recent quantitative reviews of both neurophysiological and cognitive studies using tDCS have raised questions regarding 
its effectiveness to induce reliable neuroplastic changes that measurably affect cognition in neurotypical or patient populations (Horvath et al., 2014, 2015a,b). Additional concerns pertain to the replicability of the findings reported in the existing literature and the specification of the precise conditions under which positive tDCS effects can be obtained (Mancuso et al., 2016). Overall, there is a great deal of variability in the robustness of tDCS-linked cognitive outcomes that may be largely attributed to small and heterogeneous sample sizes, the scarcity of data on dose-response effects, and substantial methodological diversity across laboratories. These limitations are exacerbated by the aforementioned lack of understanding of the precise mechanistic effects of a given tDCS protocol on the brain over short- and long-timeframes and in the context of particular tasks.

As researchers using tDCS, we are acutely aware of the limitations in interpretation and application imposed by these gaps in knowledge about the procedure and we are highly motivated to fill them. To satisfy this goal, this Frontiers Research Topic brings together 11 articles from leading experts in the field of non-invasive brain stimulation that aim to address several of the above-mentioned questions associated with tDCS, as well as examine the strength of the evidence regarding the potential of $\mathrm{tDCS}$ to modulate different aspects of cognition.

The resulting collection of articles is divided into two clusters centered around (a) methodological issues and perspectives and (b) the influence of individual and group differences in guiding tDCS effects. The first part of the E-book begins with a review by Esmaeilpour et al. of the outcomes of human trials using tDCS in psychiatric populations that helps situate the current literature regarding effectiveness of experimental designs and tDCS stimulation protocols. Harty et al. provide a strong rationale for use of mediation and moderation analyses when examining interactions between tDCS interventions, neural dynamics, and behavior. In turn, Minarik et al. focus on the importance of appropriate sample sizes to ensure replicability of tDCS findings, while highlighting the likelihood of overestimating effect sizes based on the published literature to date. Two additional papers in this section examine the effects of current polarity, intensity, and stimulation site for tDCS effects. Karuza et al. examine the consequences of parametric variations in current polarity and stimulation intensity for a cognitive control task, whereas Weinberger et al. review how interactions among task demands, tDCS polarity, and stimulation site can measurably enhance flexible thinking.

The second group of papers takes a sharp look at several individual and group differences factors that can determine the strength of tDCS effects, consideration of which is required

\section{REFERENCES}

Giordano, J., Bikson, M., Kappenman, E. S., Clark, V. P., Coslett, B., Hamblin, M. R., et al. (2017). Mechanisms and effects of transcranial direct current stimulation. Dose Res. 5, 1-22. doi: 10.1177/1559325816685467.

Horvath, J. C., Carter, O., and Forte, J. D. (2014). Transcranial direct current stimulation: five important issues we aren't discussing (but probably should be). Front. Syst. Neurosci. 8:2. doi: 10.3389/fnsys.2014.00002 to advance the interpretability of tDCS research. Wiegand et al. point out the essential role of genetically-determined variations in neural activity in predicting tDCS outcomes, which is particularly notable in studies of executive function. Likewise, Hsu et al. show that baseline differences in working memory performance interact with task difficulty and other state-dependent individual differences factors to determine responsiveness to tDCS. Rosen et al. similarly show that individual level of expertise determines whether anodal tDCS will enhance or impede performance in a jazz improvisation task. The last three papers highlight the importance of such individual and group differences factors for tDCS outcomes in clinical settings. Two empirical papers, one by McConathey et al. and a second by Norise et al., demonstrate how baseline measures of patient severity and task specificity can determine the efficacy of tDCS for the treatment of aphasia. Lastly, Mervis et al. review the extent of anodal or cathodal tDCS-guided improvements for different aspects of cognition in schizophrenia.

We are pleased with the breadth of topics covered in this collection and the issues addressed. Yet it is clear that each article raises a series of new questions in need of answers that will require much future research. For this goal to be achieved, it is critical to develop appropriate statistical methods and power analyses that will allow sufficient consideration of complex interactions among an extensive set of factors shown to drastically influence tDCS outcomes. Additionally, substantial work on the neurobiological mechanisms associated with tDCS effects in the brain is acutely needed. We hope this compilation will serve as a starting point for these investigations by framing the challenges and future directions for the use of tDCS that can determine its potential as a reliable method for cognitive rehabilitation, maintenance, or enhancement.

\section{AUTHOR CONTRIBUTIONS}

All authors listed have made a substantial, direct and intellectual contribution to the work, and approved it for publication.

\section{FUNDING}

EC is supported by the Advancing the Science of Imagination grant \#50696 from the Imagination Institute and Award \#23980 from the Brain and Behavior Research Foundation. MEB is supported by National Science Foundation Grants NSF OIA 1632849 and NSF OIA 1632738. MB is supported by NIH (Award\# 5R03EB017410-02, 5R21EB017510-02, 5R01MH092926-05) and DOD (Award\# FA9550-13-1-0073). 
transcranial direct current stimulation (tDCS). Brain Stimul. 8, 535-550. doi: 10.1016/j.brs.2015.01.400

Jamil, A., Batsikadze, G., Kuo, H. I., Labruna, L., Hasan, A., Paulus, W., et al. (2017). Systematic evaluation of the impact of stimulation intensity on neuroplastic after-effects induced by transcranial direct current stimulation. J. Physiol. 595, 1273-1288. doi: 10.1113/JP272738.

Kronberg, G., Bridi, M., Abel, T., Bikson, M., and Parra, L. C. (2017). Direct current stimulation modulates LTP and LTD: activity dependence and dendritic effects. Brain Stimul. 10, 51-58. doi: 10.1016/j.brs.2016.10.001

Mancuso, L. E., Ilieva, I. P., Hamilton, R. H., and Farah, M. J. (2016). Does transcranial direct current stimulation improve healthy working memory?: A meta-analytic review. J. Cogn. Neurosci. 28, 1063-1089. doi: 10.1162/jocn_a_00956

Nitsche, M. A., and Paulus, W. (2000). Excitability changes induced in the humanmotor cortex by weak transcranial direct current stimulation. J. Physiol. 527, 633-639. doi: 10.1111/j.1469-7793.2000.t01-1-00633.x

Nitsche, M. A., and Paulus, W. (2001). Sustained excitability elevations induced by transcranial DC motor cortex stimulation in humans. Neurology 57, 1899-1901. doi: 10.1212/WNL.57.10.1899

Purpura, D. P., and McMurtry, J. G. (1965). Intracellular activities and evoked potential changes during polarization of motor cortex. J. Neurophysiol. 28, $166-185$.
Rahman, A., Lafon, B., and Bikson, M. (2015). Multilevel computational models for predicting the cellular effects of noninvasive brain stimulation. Prog. Brain Res. 222, 25-40. doi: 10.1016/bs.pbr.2015.09.003

Reato, D., Rahman, A., Bikson, M., and Parra, L. C. (2010). Low-intensity electrical stimulation affects network dynamics by modulating population rate and spike timing. J. Neurosci. 30, 15067-15079. doi: 10.1523/JNEUROSCI.205910.2010

Conflict of Interest Statement: The City University of New York has patents on brain stimulation with $\mathrm{MB}$ as inventor. MB has equity in Soterix Medical Inc.

The other authors declare that the research was conducted in the absence of any commercial or financial relationships that could be construed as a potential conflict of interest.

Copyright (c) 2017 Chrysikou, Berryhill, Bikson and Coslett. This is an open-access article distributed under the terms of the Creative Commons Attribution License (CC BY). The use, distribution or reproduction in other forums is permitted, provided the original author(s) or licensor are credited and that the original publication in this journal is cited, in accordance with accepted academic practice. No use, distribution or reproduction is permitted which does not comply with these terms. 